\title{
Interface entropy in four dimensions as Calabi's diastasis on the conformal manifold
}

\author{
Kanato Goto and Takuya Okuda \\ Institute of Physics, University of Tokyo, \\ Komaba, Meguro-ku, Tokyo 153-8902, Japan \\ E-mail: kgoto@hep1.c.u-tokyo.ac.jp, takuya@hep1.c.u-tokyo.ac.jp
}

ABSTRACT: We conjecture an equality between (1) the entropy associated with a Janus interface in a $4 \mathrm{~d} \mathcal{N}=2$ superconformal field theory and (2) Calabi's diastasis, a particular combination of analytically continued Kähler potentials, on the conformal manifold (moduli space) of the $4 d$ theory.

KEywords: Supersymmetric Gauge Theory, Conformal Field Theory, Extended Supersymmetry

ARXiv EPrint: 1805.09981 
A Janus interface is a codimension-one object across which coupling constants take different values. The original construction in $4 \mathrm{~d}[1,2]$ did not preserve supersymmetry. This paper concerns half BPS Janus interfaces in $4 \mathrm{~d} \mathcal{N}=4[3]$ and $\mathcal{N}=2$ [4] superconformal field theories.

It is known [5] that the interface entropy, or the $g$-factor [6], of the Janus interface in $2 \mathrm{~d} \mathcal{N}=(2,2)$ theories is given by a specific combination of analytically continued Kähler potentials, namely, Calabi's diastasis [7]. It is natural to ask if a similar statement holds in other dimensions.

Recall that the interface entropy in $2 \mathrm{~d}$ admits an interpretation as a contribution of the interface to the entanglement entropy for the entangling region containing the interface [8].

The authors of [9] defined the contribution of an interface (called "defect" by them) to the entanglement entropy as follows. Let us consider the $4 \mathrm{~d}$ Minkowski space with coordinates $x^{\mu}(\mu=0, \ldots, 3)$, together with a conformal interface located at $x^{3}=0$. We have $\mathrm{CFT}_{+}\left(\mathrm{CFT}_{-}\right)$in the region $x^{3}>0\left(x^{3}<0\right)$. Take as the entangling region the round two-sphere of radius $R$ centered at the origin, and denote the resulting entanglement entropy by $S$. Let $S_{ \pm}$be the entanglement entropy defined by the same geometry, but without an interface, for $\mathrm{CFT}_{ \pm}$respectively. They define

$$
S_{\text {interface }}=S-\frac{S_{+}+S_{-}}{2} .
$$

In this combination contributions from the bulk region are canceled. Let $\epsilon$ be a UV cut-off. By a holographic analysis they found the behavior

$$
S_{\text {interface }}=D_{1} \frac{R}{\epsilon}+D_{0}+\mathcal{O}(\epsilon) .
$$

They showed $D_{1}$ to be scheme dependent, but $D_{0}$ scheme independent and thus universal.

For the Janus interface, the quantity $D_{0}$ is a non-holomorphic function $D_{0}\left(\tau, \bar{\tau} ; \tau^{\prime}, \bar{\tau}^{\prime}\right)$ that depends on two points $\tau$ and $\tau^{\prime}$ on the conformal manifold. These points correspond to the values of the couplings on the two sides of the interface. The function is subject to some constraints. First, it has to vanish when the two points coincide, i.e., when the interface is trivial. Second, it has to be invariant under S-duality transformations.

Another intriguing fact is that the sphere partition functions of $2 \mathrm{~d} \mathcal{N}=(2,2)$ and $4 \mathrm{~d}$ $\mathcal{N}=2$ SCFTs both compute the Kähler potential $K$ of the respective conformal manifold [10-12]. With a suitable normalization of the metric, the relation in the $4 \mathrm{~d}$ case reads

$$
Z_{S^{4}}=\left(\frac{r}{r_{0}}\right)^{-4 a} e^{K / 12}
$$

where $a$ is an anomaly coefficient, $r$ is the radius, and $r_{0}$ is a renormalization scale.

Any scheme-independent physical quantity associated with a Janus interface should be invariant under duality transformations when there is no globally defined Kähler potential [13]. Calabi's diastasis (the expression in the bracket of the formula below) possesses 
this property because it is invariant under Kähler transformations. This makes it a very natural function to enter the universal part of the interface entropy.

These considerations motivate us to make the following conjecture.

There exists an appropriate notion of interface entropy $D_{0}$ generalizing the definition above such that for a half BPS Janus interface in a $4 d \mathcal{N}=2$ superconformal field theory $D_{0}$ is proportional to Calabi's diastasis on the conformal manifold:

$$
D_{0}=c_{0}\left[K(\tau, \bar{\tau})+K\left(\tau^{\prime}, \bar{\tau}^{\prime}\right)-K\left(\tau, \bar{\tau}^{\prime}\right)-K\left(\tau^{\prime}, \bar{\tau}\right)\right],
$$

where $c_{0}$ is a constant.

The paper [9] applied the holographic formula [14] for entanglement entropy to the supergravity background of [15] dual to the half BPS Janus interface in $4 \mathrm{~d} \mathcal{N}=4 \mathrm{SU}(N)$ super Yang-Mills theory. Their result for $\theta^{+}=\theta^{-}$, found in equation (3.72) of their paper, is that

$$
D_{0}=-\frac{N^{2}}{2} \log \left(1+\frac{\left(g_{\mathrm{YM}}^{+}-g_{\mathrm{YM}}^{-}\right)^{2}}{2 g_{\mathrm{YM}}^{+} g_{\mathrm{YM}}^{-}}\right),
$$

where $g_{\mathrm{YM}}^{ \pm}$and $\theta^{ \pm}$are the values of the Yang-Mills coupling and the theta parameter on the two sides of the interface. Although this formula is written for the special case $\theta^{+}=\theta^{-}$, the result for the general case where both $g_{\mathrm{YM}}$ and $\theta$ vary across the interface can be obtained by an action of $\operatorname{SL}(2, \mathbb{R})$. This group is a symmetry of type IIB supergravity and transforms the dilaton and the RR scalar that are related to CFT parameters as $C_{(0)}=\theta / 2 \pi, e^{-2 \phi}=4 \pi / g_{\mathrm{YM}}^{2}$. Then the result (5) of [9] can be summarized by saying that $c_{0}=-1 / 24$ with

$$
K=-6 N^{2} \log i(\bar{\tau}-\tau), \quad \tau=\frac{\theta}{2 \pi}+i \frac{4 \pi}{g_{\mathrm{YM}}^{2}}
$$

in the large $N$ limit. The Kähler potential (6) gives the usual metric of constant negative curvature on the upper half plane, which is known to be the Zamolodchikov metric of the theory [16]. The normalization of $K$ is determined by the relation (3) and the coupling dependence $Z_{S^{4}} \propto g_{\mathrm{YM}}^{N^{2}-1}$ [17]. This provides a modest check of our conjecture.

The appearance of $N^{2}(\sim$ central charge) in (5) is similar to the situation of [18], where the holographic computation of the interface entropy for certain 2d CFT's yielded the product of the central charge and Calabi's diastasis on the moduli space of the dual supergravity.

More study is desired to check, prove, or generalize the conjecture. For a further check one may construct the holographic dual of the Janus interface for class $\mathrm{S}$ theories by deforming supergravity solutions of [19]. Does a relation similar to (4) hold for other quantities such as those characterizing reflection/transmission? We leave these matters for the future.

\section{Acknowledgments}

The research of KG is supported in part by the JSPS Research Fellowship for Young Scientists. The research of TO is supported in part by the JSPS Grants-in-Aid for Scientific 
Research No. 16K05312. TO thanks C. Bachas, E. D'Hoker, and T. Nishioka for useful discussion and correspondence. TO also acknowledges the Galileo Galilei Institute for Theoretical Physics for the hospitality and the INFN for partial support during the completion of this work.

Open Access. This article is distributed under the terms of the Creative Commons Attribution License (CC-BY 4.0), which permits any use, distribution and reproduction in any medium, provided the original author(s) and source are credited.

\section{References}

[1] D. Bak, M. Gutperle and S. Hirano, A Dilatonic deformation of $A d S_{5}$ and its field theory dual, JHEP 05 (2003) 072 [hep-th/0304129] [INSPIRE].

[2] A.B. Clark, D.Z. Freedman, A. Karch and M. Schnabl, Dual of the Janus solution: An interface conformal field theory, Phys. Rev. D 71 (2005) 066003 [hep-th/0407073] [INSPIRE].

[3] D. Gaiotto and E. Witten, Janus Configurations, Chern-Simons Couplings, And The theta-Angle in $N=4$ Super Yang-Mills Theory, JHEP 06 (2010) 097 [arXiv:0804.2907] [INSPIRE].

[4] N. Drukker, D. Gaiotto and J. Gomis, The Virtue of Defects in $4 D$ Gauge Theories and 2D CFTs, JHEP 06 (2011) 025 [arXiv: 1003.1112] [INSPIRE].

[5] C.P. Bachas, I. Brunner, M.R. Douglas and L. Rastelli, Calabi's diastasis as interface entropy, Phys. Rev. D 90 (2014) 045004 [arXiv:1311.2202] [InSPIRE].

[6] I. Affleck and A.W.W. Ludwig, Universal noninteger 'ground state degeneracy' in critical quantum systems, Phys. Rev. Lett. 67 (1991) 161 [INSPIRE].

[7] E. Calabi, Isometric imbedding of complex manifolds, Ann. Math. 58 (1953) 1.

[8] P. Calabrese and J.L. Cardy, Entanglement entropy and quantum field theory, J. Stat. Mech. 0406 (2004) P06002 [hep-th/0405152] [INSPIRE].

[9] J. Estes, K. Jensen, A. O'Bannon, E. Tsatis and T. Wrase, On Holographic Defect Entropy, JHEP 05 (2014) 084 [arXiv: 1403.6475] [INSPIRE].

[10] H. Jockers, V. Kumar, J.M. Lapan, D.R. Morrison and M. Romo, Two-Sphere Partition Functions and Gromov-Witten Invariants, Commun. Math. Phys. 325 (2014) 1139 [arXiv: 1208.6244] [INSPIRE].

[11] J. Gomis and S. Lee, Exact Kähler Potential from Gauge Theory and Mirror Symmetry, JHEP 04 (2013) 019 [arXiv: 1210.6022] [INSPIRE].

[12] E. Gerchkovitz, J. Gomis and Z. Komargodski, Sphere Partition Functions and the Zamolodchikov Metric, JHEP 11 (2014) 001 [arXiv:1405.7271] [INSPIRE].

[13] R. Donagi and D.R. Morrison, Conformal field theories and compact curves in moduli spaces, JHEP 05 (2018) 021 [arXiv:1709.05355] [INSPIRE].

[14] S. Ryu and T. Takayanagi, Holographic derivation of entanglement entropy from AdS/CFT, Phys. Rev. Lett. 96 (2006) 181602 [hep-th/0603001] [INSPIRE].

[15] E. D'Hoker, J. Estes and M. Gutperle, Exact half-BPS Type IIB interface solutions. I. Local solution and supersymmetric Janus, JHEP 06 (2007) 021 [arXiv:0705.0022] [INSPIRE]. 
[16] K. Papadodimas, Topological Anti-Topological Fusion in Four-Dimensional Superconformal Field Theories, JHEP 08 (2010) 118 [arXiv:0910.4963] [INSPIRE].

[17] V. Pestun, Localization of gauge theory on a four-sphere and supersymmetric Wilson loops, Commun. Math. Phys. 313 (2012) 71 [arXiv:0712. 2824] [InSPIRE].

[18] E. D'Hoker and M. Gutperle, Holographic entropy and Calabi's diastasis, JHEP 10 (2014) 093 [arXiv: 1406.5124] [INSPIRE].

[19] D. Gaiotto and J. Maldacena, The Gravity duals of $N=2$ superconformal field theories, JHEP 10 (2012) 189 [arXiv:0904.4466] [INSPIRE]. 